\title{
Presumed Pyogenic Granuloma Associated with Intravitreal Anti- Vascular Endothelial Growth Factor Therapy
}

\author{
Jesse J. Jung ${ }^{1,2}$, Kara E. Della Torre ${ }^{1,2,3}$, Millie R. Fell ${ }^{1}$, Christopher C. Teng ${ }^{1,4}$ and K. Bailey Freund ${ }^{*}, 1,2,3,5$ \\ ${ }^{I}$ Department of Ophthalmology, New York University School of Medicine, New York, NY, USA \\ ${ }^{2}$ LuEsther T. Mertz Retinal Research Center, Manhattan Eye, Ear and Throat Institute, New York, NY, USA \\ ${ }^{3}$ Vitreous, Retina, Macula Consultants of New York, New York, NY, USA \\ ${ }^{4}$ Einhorn Clinical Research Center, The New York Eye and Ear Infirmary, New York, NY, USA \\ ${ }^{5}$ Edward S. Harkness Eye Institute, Columbia University College of Physicians and Surgeons, New York, NY, USA
}

\begin{abstract}
To report a case of a presumed pyogenic granuloma at the site of multiple intravitreal anti-Vascular Endothelial Growth Factor (VEGF) injections. Intravitreal anti-VEGF injections can be complicated by a localized reaction of the conjunctiva.
\end{abstract}

Keywords: Ranibizumab (Lucentis), age-related macular degeneration, pyogenic granuloma, nodule, inflammation.

\section{INTRODUCTION}

Age-related macular degeneration (AMD) is one of the most common causes of severe, irreversible vision loss in older adults in developed countries. Neovascular AMD is characterized by abnormal growth of new blood vessels under or within the macula [1]. Vascular endothelial growth factor A (VEGF) is a key modulator of angiogenesis and promotes neovascularization in AMD [1-4]. Currently, several anti-VEGF drugs such as pegaptanib, ranibizumab and bevacizumab are used via the intravitreal route for treatment of neovascular AMD [5].

Ranibizumab (Lucentis, Genentech, San Francisco, USA) is a recombinant humanized monoclonal $\mathrm{G}_{1 \mathrm{~K}}$ isotype antibody Fab with anti-angiogenetic VEGF A blocking properties [1-4]. The Food and Drug Administration (FDA) approved Ranibizumab since 2006 as an intravitreal injection treatment for neovascular AMD [1,5]. Bevacizumab (Avastin, Genentech, San Francisco, USA) is a recombinant humanized full-length antibody that binds to all isoforms of VEGF. It was initially approved by the FDA for intravenous use against metastatic colorectal cancer, but is now commonly used off-label to treat a variety of retinal disorders including neovascular AMD, diabetic macular edema, and retinal vein occlusion [7].

Several adverse reactions after intravitreal injections of anti-VEGF treatment have been reported including subconjunctival hemorrhage [4-7], conjunctival hyperemia [4-7], transient and/or sustained elevated intraocular pressure (IOP) $[1,4-9]$, anterior uveitis $[1,8-10]$, iatrogenic cataract $[1,5]$, vitreous hemorrhage $[1,4]$, retinal tear or detachment $[1,4]$, central retinal vein occlusion [4] and endophthalmitis

*Address correspondence to this author at the 460 Park Avenue, Fifth Floor, New York, NY 10022, USA; Tel: 212-861-9797; Fax: 212-628-0698;

E-mail: kbfnyf@aol.com
$[1,4]$. We report a case of a localized, subconjunctival, nodular inflammatory reaction at the site of multiple ranibizumab injections. Based on clinical appearance, anterior segment imaging and improvement with topical steroid treatment, we believe this lesion to be a pyogenic granuloma. To the best of our knowledge, this is the first case of pyogenic granuloma observed following intravitreal injections of anti-VEGF therapy.

\section{CASE REPORT}

An 87-year-old Caucasian man treated for neovascular AMD presented complaining of eye pain and redness one week after his last intravitreal ranibizumab injection in the right eye (Fig. 1a). The patient received OCT guided treatment with bevacizumab $(0.125 \mathrm{mg} / 0.05 \mathrm{ml})$ starting in October 2005 prior to Federal Drug Administration (FDA) approval of ranibizumab for AMD treatment and received three bevacizumab $(0.125 \mathrm{mg} / 0.05 \mathrm{ml})$ over an 8 month period of time. He elected to transition to a treat and extend protocol after FDA approval of ranibizumab for treatment of neovascular AMD and received twenty-one ranibizumab $(0.5 \mathrm{mg} / 0.05 \mathrm{ml})$ injections without complications starting August 2006 and continued over a 48 month period time with his last injection on August 2010. All injections were administered $3.5-4 \mathrm{~mm}$ posterior to the limbus in the inferotemporal quadrant varying one or two clock hours from previous injection sites using a 32 gauge needle following three drops of topical proparacaine $(0.5 \%)$, two drops of ofloxacin $(0.3 \%)$, and one drop of $5 \%$ povidoneiodine. According to the intravitreal injection protocol recommended in the literature, prior to each injection, the injection site was dried with a sterile cotton-tipped applicator. A wire lid speculum and non-sterile powder-free latex gloves were used for all injection procedures [11].

One week post intravitreal injection, the patient had stable corrected visual acuity of 20/200 in the affected right eye. There was localized, inferotemporal hyperemia of the 
bulbar conjunctiva at the site of the injection. Within the area of hyperemia there was an elevated, subconjunctival erythematous nodule, which was slightly mobile when depressed with a cotton tip applicator (Fig. 1b). There was no evidence of perilimbal vessel injection. On eyelid eversion, there was no tarsal or forniceal conjunctival involvement or foreign body. Anterior exam revealed trace cells and 1+ flare reaction in the anterior chamber. No hypopyon or vitreous cells were noted. Lens, optic nerve and peripheral retinal exam were unremarkable. The left eye was unremarkable and showed no evidence of inflammation.

(1A)

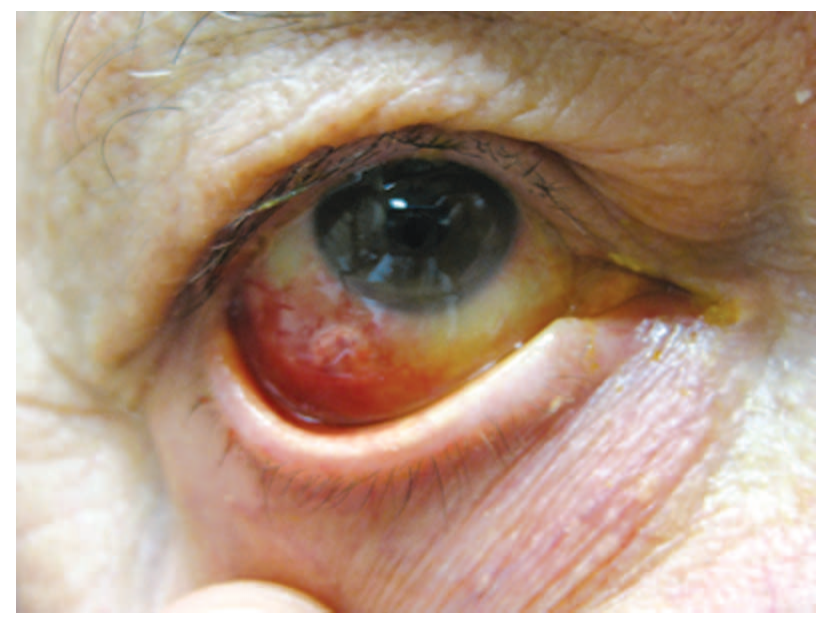

(1B)

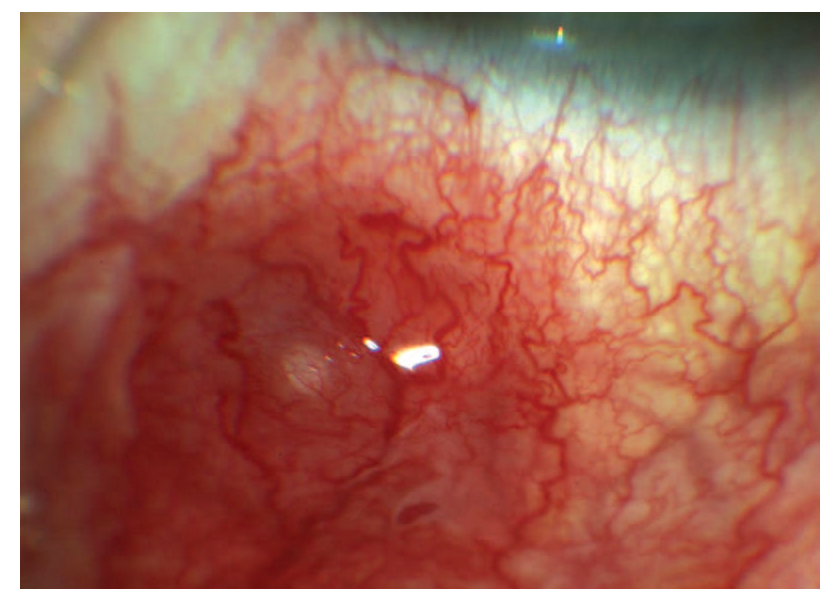

Fig. (1). (A) Color photograph shows the appearance of the right eye at presentation. There is hyperemia and edema of the inferotemporal bulbar conjunctiva. (B) Higher magnification of initial erythematous subconjunctival nodule at the site of prior intravitreal injection.

Slit lamp optical coherence tomography (SL-OCT) (Fig. 2a) and ultrasound biomicroscopy (UBM) (Fig. 2b) revealed a well-circumscribed, solid lesion without scleral penetration. The patient was started on ofloxacin $(0.3 \%)$ and rimexolone $(1 \%)$ four times per day in the right eye. Two weeks post-injection, the patient self-discontinued the ofloxacin drops and was using only rimexolone twice daily. While the nodule persisted and conjunctival hyperemia had improved minimally, the patient reported little discomfort.
He was transitioned to prednisolone acetate (1\%) four times per day and fluorometholone ointment $(0.1 \%)$ at bedtime. The lesion decreased in size slowly over five months and prednisolone acetate was slowly tapered to one drop daily. The hyperemia resolved and there was trace hyperpigmentation over the area of minimal subconjunctival elevation (Fig. 3). Surgical excision and biopsy was deferred due to improvement with the topical steroid. The patient's AMD treatment was changed to pro re nata (PRN) optical coherence tomography guided dosing and has not since required or received any further injections. If clinically indicated, another injection site will be considered to prevent further complications.

\section{DISCUSSION}

Safety profiles are closely monitored in the original clinical trials of a medication, but long term use of a medication can subsequently lead to the new presentation of adverse events or drug reactions [2]. The most common ocular adverse events for patients receiving ranibizumab in randomized clinical trials were transient subconjunctival hemorrhage [4-7], intraocular inflammation [1,8-10] and transient and/or sustained elevated intraocular pressure (IOP) [1,4-9]. In one prospective study monitoring for transient post injection IOP elevations after ranibizumab injections, the authors noted that mild injection site reactions such as subconjunctival hemorrhage and /or hyperemia were common $(59.2 \%)$ [6].

Transient ischemia of the ocular surface after intravitreal bevacizumab has been reported with nonperfusion of conjunctival, episcleral and scleral vessels around the injection site. This ischemia, hypothesized to be inflammatory in nature, was treated with topical steroids and resolved over three weeks [12]. Our case did not show any signs of nonperfusion; rather, hyperemia of the blood vessels overlying the injection site was seen.

Intravitreal ranibizumab injections have rarely been associated with noninfectious anterior uveitis [1,8-10]. Within one week after injection, our patient developed active anterior inflammation and a localized inflammatory reaction at the site of intravitreal injection. He had previously tolerated multiple bevacizumab and ranibizumab treatments without evidence of post-injection inflammation.

The differential diagnosis at presentation included foreign body reaction, scleral abscess, nodular scleritis, and pyogenic granuloma. Anterior imaging with SL-OCT and UBM revealed a solid nodule that did not involve the sclera, which was consistent with a localized reaction. We believe this reaction occurred as a result of inflammatory materials deposited in the process of injection, creating a pyogenic granuloma reaction and secondary anterior chamber inflammation.

Pyogenic granulomas are red colored tumors that appear on the palpebral or bulbar conjunctiva in areas of vascularized tissue submitted to chronic inflammatory injury [13-19]. Histopathologically, they contain granulation tissue with blood vessels arranged in a fan-shaped configuration and have an associated acute and chronic inflammatory cell infiltrate in a loose connective tissue stroma [15,16]. Although often associated with trauma [14-16], surgery [1417] and chalazions [13-16], there are reports of pyogenic 


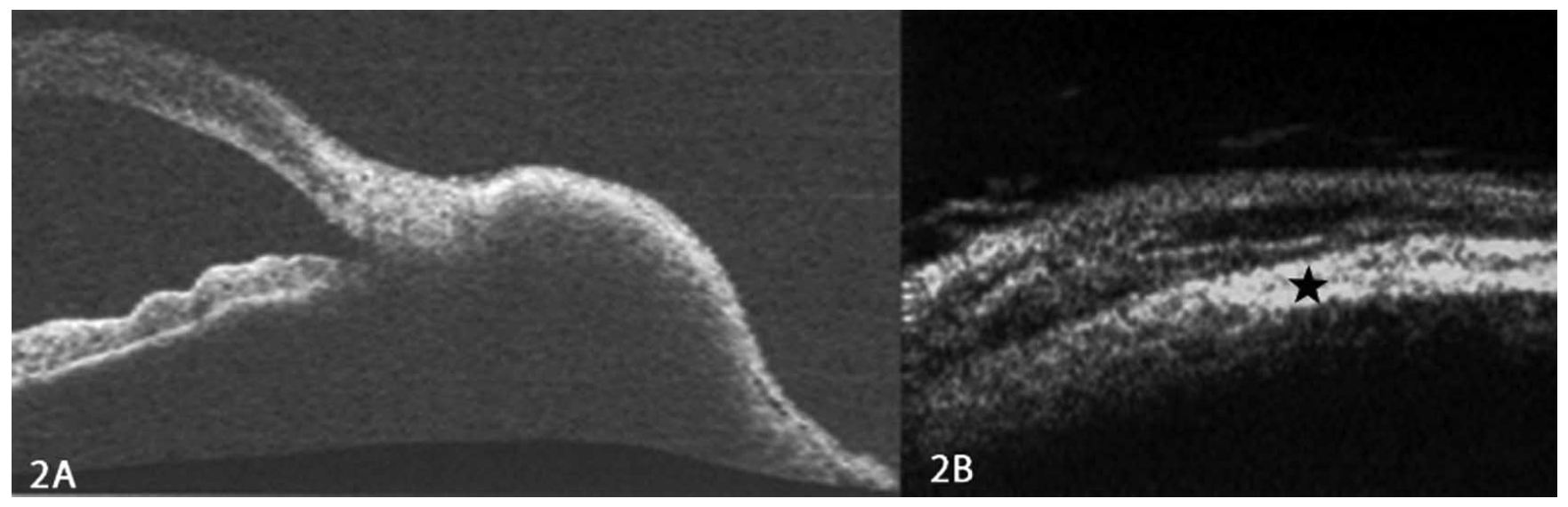

Fig. (2). (A) Anterior slit lamp optical coherence tomography imaging showing a well-circumscribed nodule. (B) Ultrasound biomicroscopy showing no penetration of the normal sclera (black star).

granuloma formation after foreign bodies such as natural or synthetic fibers [16], contact lens [16], scleral buckle [14], and insect wing [14]. We hypothesize that a component of the intravitreal injection procedure such as talc from a disposable glove, cotton fibers from the sterile applicator, or silicone oil derived from the syringe [20-22] could have triggered foreign body reaction at the level of the conjunctiva. Also, repeated injections in the same quadrant of the eye might have increased the risk of this complication.

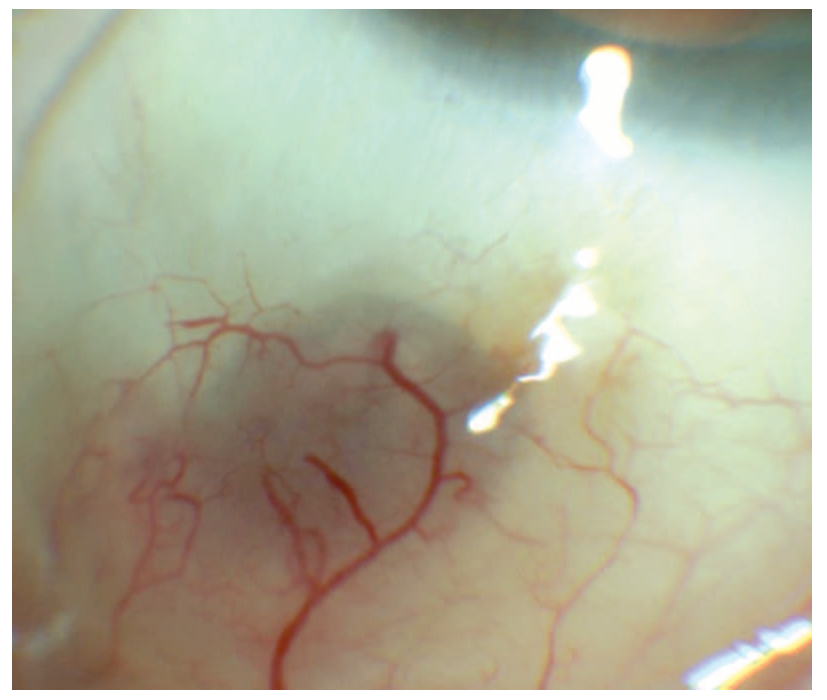

Fig. (3). Color photograph showing the appearance following 5 months topical steroid treatment. The conjunctival nodule has flattened leaving persistent dilated episcleral vessels and increased scleral translucency.

Intravitreal anti-VEGF agents including ranibizumab and bevacizumab are a safe and effective treatment for neovascular AMD [5]. Even with the evidence and safety profiles available from the original clinical trials, new presentations of adverse events may occur as the usage of intravitreal anti-VEGF therapy continues to grow. The safety data on these treatment modalities will need to be continuously monitored and the potential risks and benefits of intravitreal anti-VEGF agents should be discussed with patients receiving treatment for neovascular AMD [9].

\section{CONFLICTS OF INTEREST}

Financial support from the LuEsther T. Mertz Retinal Research Center, Manhattan Eye, Ear, and Throat Institute, and The Macula Foundation Inc. The funding organizations had no role in the design or conduct of this research.

Financial disclosure includes Genentech (Consultant, Research Support-KBF), Alcon (Consultant-KBF) and Alimera (Consultant-KBF).

\section{REFERENCES}

[1] Rosenfeld PJ, Brown DM, Heier JS, et al. Ranibizumab for neovascular age-related macular degeneration. N Engl J Med 2006; 355: 1419-31.

[2] Chen Y, Wiesmann C, Fuh G, et al. Selection and analysis of an optimized anti-vegf antibody: crystal structure of an affinitymatured fab in complex with antigen. J Mol Bio 1999; 293: 865-81.

[3] Dafer RM, Schneck M. Intravitreal ranibizumab and bevacizumab: a review of risk. Semin Ophthalmol 2007; 22: 201-4.

[4] Heier JS, Antoszyk AN, Pavan PR, et al. Ranibizumab for treatment of neovascular age-related macular degeneration a phase I/II multicenter, controlled, multidose study. Ophthalmol 2006; 113: $633-42$

[5] Jeganathan VSE and Verma N. Safety and efficacy of intravitreal anti-VEGF injections for age-related macular degeneration. Curr Opin Ophthalmol 2009; 20: 223-5.

[6] Gismondi M, Salati C, Salvetat ML, et al. Short-term effect of intravitreal injection of ranibizumab (lucentis) on intraocular pressure. J Glaucoma 2009; 18: 658-61.

[7] Tseng JJ, Vance SK, Della Torre KE, et al. Sustained increased intraocular pressure related to intravitreal anti-vascular endothelial growth factor therapy for neovascular age-related macular degeneration. J Glaucoma 2011; Epub.

[8] Ladas ID, Karagiannis DA, Rouvas AA, et al. Safety of repeat intravitreal injections of bevacizumab versus ranibizumab our experience after 2,000 injections. Retina 2009; 29: 313-8.

[9] Jager RD, Aiello LP, Patel SC, et al. Risks of intravitreous injection: a comprehensive review. Retina 2004; 24: 676-98.

[10] Pieramici DJ, Avery RL, Castellarin AA, et al. Case of anterior uveitis after intravitreal injection of bevacizumab. Retina, J Retinal and Vitreous Diseases 2006; 26: 841-2.

[11] Aiello LP, Brucker AJ, Chang S, et al. Evolving guidelines for intravitreous injections. Retina 2004; 24: S3-S19.

[12] Georgakarakos ND, Sullivan P, Dowler J. Injection site ischemia and inflammation after intravitreal bevacizumab. Retin Cases Brief Rep 2010; 4: 294-6.

[13] D'Hermies F, Meyer A, Morel X, et al. Conjunctiva pyogenic granuloma in a patient with chalazions. French J Ophthalmol 2003; 26:1085-8. 
[14] Damasceno EF, Pereira C, Damasceno NAP, et al. Pyogenic granuloma after retinal detachment surgery with scleral buckle: case report. Arq Bras Oftalmol 2009; 72: 543-4.

[15] Grossniklaus H, Green WR, Luckenbach M, Chan CC. Conjunctival Lesions in adults. a clinical and histopathologic review. Cornea 1987; 6: 78-116.

[16] Ferry AP. Pyogenic granulomas of the eye and ocular adnexa: a study of 100 cases. Trans Am Ophthalmol Soci 1989; 87: 327-43.

[17] Googe JM, Mackman G, Peterson MR. Pyogenic granulomas of the cornea. Survey Ophthalmol 1984; 29: 188-92.
[18] Horton JC, Mathers WD, Zimmerman LE. Pyogenic Granuloma of the palpebral conjunctiva associated with contact lens wear. Cornea 1990; 9: 359-61.

[19] Venkatesh P, Lakshmaiah NC, Chawla R. Insect wing conjunctival granuloma. Cornea 2003; 22: 489-90.

[20] Freund KB, Laud K, Eandi CM, Spaide RF. Silicone oil droplets following intravitreal injection. Retina 2006; 26: 701-3.

[21] Bakri SJ, Ekdawi NS. Intravitreal silicone oil droplets after intravitreal drug injections. Retina 2008; 28: 996-1001.

[22] Kocabora MS, Ozbilen KT, Serefoglu K. Intravitreal silicone oil droplets following pegaptanib injection. Acta Ophthalmol 2010; 88: e44-5.

(C) Jung et al.; Licensee Bentham Open.

This is an open access article licensed under the terms of the Creative Commons Attribution Non-Commercial License (http: //creativecommons.org/licenses/by$\mathrm{nc} / 3.0 /$ ) which permits unrestricted, non-commercial use, distribution and reproduction in any medium, provided the work is properly cited. 